\title{
ESTIMATIVA DO CONSUMO MÁXIMO DE OXIGÊNIO E ANÁLISE DE CONCORDÂNCIA ENTRE MEDIDA DIRETA E PREDITA POR DIFERENTES TESTES DE CAMPO
}

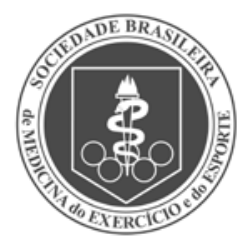

Artigo Original

\author{
ESTIMATED MAXIMUM OXYGEN UPTAKE AND AGREEMENT ANALYSIS BETWEEN DIRECT \\ AND PREDICTED MEASUREMENT BY DIFFERENT FIELD TESTS \\ ESTIMATIVA DEL CONSUMO MÁXIMO DE OXÍGENO Y ANÁLISIS DE CONCORDANCIA ENTRE \\ MEDIDA DIRECTA Y PREVISTA, SEGÚN DIVERSAS PRUEBAS DE CAMPO
}

\author{
Mariana Biagi Batista \\ (Educador Físico) $)^{1,2}$ \\ Edilson Serpeloni Cyrino \\ (Educador Físico) $)^{1,2}$ \\ Vinícius Flávio Milanez \\ (Educador Físico) $^{3}$ \\ Manuel João Coelho e Silva \\ (Educador Físico) 1,2,5 \\ Miguel de Arruda \\ (Educador Físico) 1,4 \\ Enio Ricardo Vaz Ronque \\ (Educador Físico) ${ }^{1,2}$
}

1. Grupo de Estudo e Pesquisa em Atividade Física e Exercício. Centro de Educação Física e Esporte. Universidade Estadual de Londrina, PR, Brasil.

2. Grupo de Estudo e Pesquisa em Metabolismo, Nutrição e Exercício. Centro de Educação Física e

Esporte. Universidade Estadual de Londrina, PR, Brasil.

3. Centro de Educação Física. Universidade Estadual de

Londrina, PR, Brasil.

4. Faculdade de Educação Física. Universidade Estadual de Campinas, PR, Brasil.

5. Faculdade de Ciência do Desporto e Educação Física. Universidade de Coimbra, Portugal.

\section{Correspondência:}

Enio Ricardo Vaz Ronque Grupo de Estudo e Pesquisa em Atividade Física e Exercício GEPAFE - Centro de Educação Física e Esporte, Universidade Estadual de Londrina. Rodovia Celso Garcia Cid, km 380, Campus Universitário. 86051-990 - Londrina, PR, Brasil. enioronque@uel.br

\section{RESUMO}

Objetivo: Verificar a estimativa da potência aeróbia e a concordância entre a medida direta e predita por três diferentes testes de campo em jovens universitários. Métodos: Participaram do estudo 12 sujeitos (23,1 2,8 anos), sete homens e cinco mulheres, que foram submetidos a medidas antropométricas de massa corporal, estatura e espessura de dobras cutâneas. Além disso, os sujeitos realizaram um teste máximo direto (MD) em esteira e três testes de campo para verificação do consumo máximo de oxigênio $\left(\mathrm{VO}_{2 \text { máx }}\right)$, em valores relativos. Os testes de campo utilizados foram: corrida/caminhada de 12 minutos de Cooper (COOPER), corrida/caminhada de uma milha (MILHA) e shuttle run de 20 metros (SR-20m). Os dados foram analisados por procedimentos descritivos e para as comparações entre a medida direta e cada um dos testes de campo foi utilizado o teste $t$ pareado. A regressão linear simples forneceu informações quanto ao desempenho dos testes e suas respectivas equações, enquanto que a concordância entre os métodos foi feita pela análise de Bland-Altman, com determinação da tendência de medida. Resultados: Não foram encontradas diferenças significantes entre o MD e os três testes de campo. Os erros padrão de estimativa variaram de aproximadamente 5,8 a 6,0 ml.kg-1 $\mathrm{min}^{-1}$, e as correlações de $r=0,61-0,64$. Os limites de concordância foram considerados amplos para os três testes, porém sem viés e tendência de estimativa. Conclusão: Apesar da similaridade entre os valores médios obtidos nas comparações entre os testes de campo e a medida de referência, o teste da MILHA foi o que apresentou melhores resultados de desempenho e concordância para a estimativa do $\mathrm{VO}_{2 \text { máx }}$

Palavras-chave: aptidão física, consumo de oxigênio, teste de esforço.

\section{ABSTRACT}

Objective: To verify the estimate of aerobic power and agreement between direct and predicted measurement by three different field tests. Methods: The study included 12 subjects (23.1 12.8 years), seven men and five women, who underwent anthropometric measurements of body mass, height and thickness of skin folds. Moreover, the subjects performed a maximal direct test (DT) in a treadmill and three field tests for verification of maximal oxygen uptake $\left(\mathrm{VO}_{2 \text { max }}\right)$, in relative values. The field tests performed were: run/walk, 12 minutes by Cooper (COOPER), run/walk a mile (MILE), and 20 meter shuttle run (SR-20M). Data were analyzed by descriptive procedures and for comparisons between the direct measurement and each of the field tests, the paired t test was used. Simple linear regression provided information about the performance of tests and their equations, while the agreement between the methods was made by Bland-Altman analysis, with determination of the t measurement trend. Results: No significant differences were found between the MD and the three field tests. The standard errors of estimation ranged from about 5.8 to $6.0 \mathrm{ml} . \mathrm{kg}^{-1} . \mathrm{min}^{-1}$ and the correlation of $r=0.61-0.64$. The limits of agreement were considered large for the three tests, but without bias and an estimation trend. Conclusions: Despite the similarity between the mean values obtained in comparisons between the field tests and the measurement of reference, the MILE test showed the best performance and agreement to estimate the $\mathrm{VO}_{2 \max }$.

Keywords: physical fitness, oxygen consumption, exercise test.

\section{RESUMEN}

Objetivo: Verificar la estimativa de la potencia aeróbica y la concordancia entre la medida directa y la prevista, según tres diferentes pruebas de campo en jóvenes universitarios. Métodos: Participaron en el estudio 12 individuos (23,1 $\pm 2,8$ años), siete hombres y cinco mujeres, quienes fueron sometidos a medidas antropométricas de masa corporal, estatura y espesura de dobleces cutáneos. Además de eso, las personas realizaron una prueba máxima directa (MD) en estera y tres pruebas de campo para verificación del consumo máximo de oxígeno $\left(\mathrm{VO}_{2 m a x x}\right)$, en valores relativos. Las pruebas de campo que se utilizaron fueron: carrera/caminata de 12 minutos de Cooper (COOPER), carrera/caminata de una milla (MILLA) [1,6 quilómetro] y shuttle run de 20 metros (SR-20m). Los datos fueron analizados mediante procedimientos descriptivos, y se utilizó la prueba ten pares para las comparaciones entre la medida directa y cada una de las pruebas de campo. La regresión linear simple suministró informaciones cuanto al desempeño en las pruebas y 
sus respectivas ecuaciones, mientras que la concordancia entre los métodos se hizo por el análisis de Bland-Altman, con determinación de la tendencia de medida. Resultados: No se encontraron diferencias significativas entre el MD y las tres pruebas de campo. Los errores estándares de estimativas variaron desde, aproximadamente, 5,8 a 6,0 ml. $\mathrm{kg}^{-1}$. $\mathrm{min}^{-1}$, y las correlaciones de $\mathrm{r}=0,61-0,64$. Los límites de concordancia fueron considerados como siendo amplios para las tres pruebas, no obstante, sin bies ni tendencia de estimativa. Conclusión: Aun considerando la similitud entre los valores promedios obtenidos en las comparaciones, entre las pruebas de campo y la medida de referencia, la prueba de la MILLA fue la que presentó mejores resultados de desempeño y concordancia para la estimativa del $V_{2} \mathrm{Záx}_{\text {. }}$

Palabras clave: aptitud física, consumo de oxígeno, prueba de esfuerzo.

Artigo recebido em 13/04/2011, aprovado em 07/10/2013.

\section{INTRODUÇÃO}

Os avanços científicos e tecnológicos têm resultado no desenvolvimento de equipamentos que possibilitam medidas e avaliações mais consistentes do desempenho físico em atletas e não atletas. Entretanto, o custo operacional relativamente elevado e a limitada aplicação, em muitas situações, têm desafiado pesquisadores e profissionais, sobretudo, das áreas de saúde e do esporte a desenvolverem e validarem testes de campo que possam proporcionar informações de boa qualidade, de maneira rápida, não invasiva ou pouco invasiva, com boa precisão, reprodutibilidade e concordância.

Nesse sentido, historicamente muitos testes de campo têm sido desenvolvidos e validados em diferentes populações para avaliação da aptidão cardiorrespiratória, uma vez que existe uma estreita relação entre o consumo máximo de oxigênio $\left(\mathrm{VO}_{2 \text { máx }}\right)$ e o desempenho atlético em diversas modalidades esportivas, bem como com a saúde, doença e mortalidade em não atletas. Neste último contexto, especificamente, existem fortes evidências de que índices elevados de aptidão cardiorrespiratória podem reduzir o risco de morbidade e mortalidade por todas as causas e por doenças cardiovasculares na população adulta ${ }^{1-3}$.

A medida (de forma direta) ou a estimativa (de forma indireta) do $V_{\mathrm{V}_{\text {máx }}}$ para avaliação da aptidão cardiorrespiratória tem sido amplamente utilizada, sobretudo, no campo da pesquisa, na área clínica e do esporte de alto rendimento, por ser reconhecidamente um dos melhores índices preditores da potência aeróbia ${ }^{4,5}$. A principal medida de referência para o $\mathrm{VO}_{2 \text { máx }}$ tem sido obtida por meio da utilização do método de calorimetria indireta. Considerando que para este tipo de medida são necessários equipamentos sofisticados e de alto custo, além da presença de técnicos e profissionais capacitados para atuarem com esse método, dificultam sua aplicação em larga escala. Adicionalmente, o fato de apenas um sujeito ser avaliado por vez prolonga o tempo das avaliações, inviabilizando a sua utilização em grandes populações ${ }^{6-8}$.

Por outro lado, a aplicação de testes de campo que proporcionam a predição do $\mathrm{VO}_{2 \text { máx }}$ por meio de modelos matemáticos é uma alternativa bastante atraente, uma vez que permite a redução dos custos operacionais e favorece a aplicação em larga escala, em ambientes diversos ${ }^{9,10}$. A grande preocupação com relação à utilização de testes de campo para essa finalidade reside na qualidade das informações produzidas, uma vez que o nível de precisão da estimativa do $\mathrm{VO}_{2 \text { máx }}$ a partir de um determinado teste pode ser comprometido pela falta de controle de diversas variáveis tais como idade, nível de aptidão física individual, sexo, entre outras. Este fato tem motivado pesquisadores a investigar a qualidade das informações produzidas por diversos testes de campo utilizados para a estimativa do $\mathrm{VO}_{2 \text { máx }}$ com base em medidas diretas obtidas em situação de campo ou laboratório por meio de equipamentos desenvolvidos, principalmente, para calorimetria indireta.

Embora parte desses estudos tenha encontrado boas correlações entre os valores estimados por testes de campo e medidos em testes de laboratório ${ }^{8,10-14}$, sobretudo em indivíduos adultos, essa informação não parece ser suficiente para avaliar a qualidade das informações produzidas, visto que a força da correlação por si só não indica uma boa concordância entre dois métodos. Desta forma, a utilização de procedimentos estatísticos mais robustos para o tratamento dos dados pode proporcionar um diagnóstico mais criterioso acerca das informações produzidas até o presente momento ${ }^{15}$.

Sendo assim, o objetivo do presente estudo foi analisar a estimativa do $\mathrm{VO}_{2 \text { máx }}$ e a concordância entre a medida direta e predita por três diferentes testes de campo em adultos jovens universitários.

\section{MÉTODOS}

\section{Sujeitos}

Doze sujeitos (sete homens e cinco mulheres), todos universitários, não atletas, na faixa etária dos 20 aos 31 anos participaram, voluntariamente, deste estudo. Todos os participantes, após receberem informações sobre as finalidades do estudo e os procedimentos aos quais seriam submetidos, assinaram um Termo de Consentimento Livre e Esclarecido. Este estudo foi desenvolvido em conformidade com as instruções contidas na Resolução 196/96 do Conselho Nacional de Saúde para estudos com seres humanos, do Ministério da Saúde.

\section{Delineamento experimental}

Na primeira visita ao laboratório os sujeitos receberam orientações gerais sobre o projeto, os procedimentos a serem adotados nas coletas e assinaram o Termo de Consentimento Livre e Esclarecido. Em seguida, medidas antropométricas foram realizadas. A ordem de execução dos quatro testes para avaliação do $\mathrm{VO}_{2 \text { máx }}$ foi determinada individualmente, de forma aleatória.

Um teste progressivo em esteira foi realizado no laboratório para determinação do $\mathrm{VO}_{2 \text { máx }}$ como medida de referência. Todas as visitas foram realizadas em intervalos míninos de $48 \mathrm{~h}$ e os sujeitos foram submetidos aos quatro protocolos de testes motores, conforme ordem previamente determinada.

\section{Antropometria}

Medidas antropométricas de massa corporal foram obtidas em uma balança da marca Filizola (Brasil), com precisão de 0,1 kg, ao passo que a estatura foi determinada em um estadiômetro de madeira com precisão de $0,1 \mathrm{~cm}$, de acordo com procedimentos padronizados descritos na literatura ${ }^{16}$.

Medidas de espessura de dobras cutâneas foram obtidas em sete pontos anatômicos (subescapular, suprailíaca, abdominal, tricipital, bicipital, coxa e perna medial), seguindo as recomendações internacionais ${ }^{17}$. Todas as medidas foram realizadas por um único avaliador, com um compasso Lange (Cambridge Scientific Instruments, Cambridge, MD), de forma rotacional. Todas as medidas foram replicadas três vezes em cada hemicorpo, sendo registrado o valor médio. O coeficiente teste-reteste excedeu 0,97 para cada um dos pontos anatômicos e o erro 
técnico de medida variou de 0,31 a 0,77 mm, não excedendo 5\% em nenhum dos pontos anatômicos medidos.

\section{Medida direta do $\mathrm{VO}_{2 \text { máx }}$}

O teste que serviu como método de referência para comparação com os resultados dos diferentes testes de campo foi realizado em laboratório em uma esteira elétrica (ATL 10200, Inbrasport, Porto Alegre, Rio Grande do Sul, RS, Brasil), em ambiente com temperatura média controlada de $23^{\circ} \mathrm{C}$, utilizando-se o protocolo de Bruce modificado. Para medida direta do $\mathrm{VO}_{2 \text { máx }}$ foi utilizado o sistema ergoespirométrico computadorizado (VO-2000, Aerosport, Medgraphics, St. Paul, Minnesota, EUA). A calibração do analisador de gases foi realizada pelo próprio aparelho durante o período de uma hora antes do início da primeira avaliação, seguindo as recomendações do fabricante. As amostras gasosas foram coletadas e mensuradas a cada 10 s durante o teste.

A frequência cardíaca foi acompanhada a cada minuto com o uso do monitor da marca Polar ${ }^{\circledR}$ (Polar Electro OY, Finlândia), modelo S810i. Para a determinação do $\mathrm{VO}_{2 \text { máx }}$ foram utilizados como critérios os valores de: a) quociente respiratório superior a 1,1 ; b) $\mathrm{VO}_{2}$ constante ou com variações inferiores a 2,1 $\mathrm{ml} \mathrm{O}_{2} \cdot \mathrm{kg}^{-1} \cdot \mathrm{min}^{-1}$ ou $150 \mathrm{ml} \mathrm{O}$. $\mathrm{min}^{-1}$ nos últimos dois minutos do teste com aumento de carga; $c$ ) frequência cardíaca do teste maior que 90\% da frequência cardíaca máxima estimada pela equação 220-idade. Assim, o maior valor de $\mathrm{VO}_{2}$ obtido foi considerado o $\mathrm{VO}_{2 \text { máx}}$.

\section{Testes de campo para estimativa do $\mathrm{VO}_{2 \text { máx }}$}

O teste de corrida/caminhada de 12 min (COOPER) foi realizado em uma pista oficial de atletismo (400 m). Os sujeitos foram orientados a percorrer a máxima distância possível no tempo de 12 min. Para a estimativa do $\mathrm{VO}_{2 \text { máx }}\left(\mathrm{ml} \mathrm{kg}^{-1} \cdot \mathrm{min}^{-1}\right)$ foi utilizada uma equação ${ }^{18}$ baseada na máxima distância percorrida (DP): $\mathrm{VO}_{2 \text { máx }}=(\mathrm{DP}-504,1) / 44,79$.

O teste de corrida/caminhada de uma milha (MILHA) foi realizado na mesma pista oficial de atletismo (400 m). Os sujeitos foram orientados a percorrer a distância predeterminada, no mínimo tempo possível. Para a estimativa do $\mathrm{VO}_{2 \text { máx }}\left(\mathrm{ml} \mathrm{kg}^{-1} \cdot \mathrm{min}^{-1}\right)$ adotou-se uma equação ${ }^{19}$ baseada no tempo total (T) despendido em minutos: $\mathrm{VO}_{2 \text { máx }}=100,311$ $-(9,602) \times T+(0,287) \times T^{2}$.

O teste de corrida de vai-e-vem de $20 \mathrm{~m}$ (SR-20m) foi executado em uma quadra poliesportiva de um ginásio coberto. Os procedimentos para realização do teste, bem como os critérios de interrupção seguiram as recomendações de Léger e Lambert ${ }^{20}$. A velocidade (V) em km/h no último estágio completado pelo avaliado foi utilizada para a estimativa do $\mathrm{VO}_{2 \text { máx }}\left(\mathrm{ml}^{\mathrm{kg}} \mathrm{kg}^{-1} \cdot \mathrm{min}^{-1}\right)$, de acordo com a equação ${ }^{11}$ : $\mathrm{VO}_{2 \text { máx }}=-27,4+6,0 \times \mathrm{V}$.

\section{Tratamento estatístico}

Após a confirmação da normalidade dos dados por meio do teste de Shapiro-Wilk, as informações foram tratadas por procedimentos paramétricos (média, desvio padrão, valores mínimos e máximos). O teste $t$ pareado foi utilizado nas comparações entre os escores de $\mathrm{VO}_{2 \text { máx }}$ estimados pelos testes de campo COOPER, MILHA e SR-20M e a medida direta obtida em teste de laboratório.

Análises de regressão simples foram utilizadas adotando-se a medida direta do $\mathrm{VO}_{2 \text { máx }}$ como variável dependente e as estimativas geradas por cada teste de campo, como variáveis independentes. Os valores de slope e intercepto, produzidos a partir das análises de regressão, foram comparados com a linha de identidade.

O erro padrão de estimativa (EPE) e o coeficiente de correlação de Pearson (r) foram analisados. O EPE foi utilizado como uma medida de validação para analisar a falta de associação entre a medida direta do
$V_{\mathrm{O}_{2 m a ́ x}}$ e as medidas estimadas por cada teste de campo. O erro puro (EP) também foi avaliado como outra medida de validação utilizando a seguinte equação $\left(\Sigma\left(Y_{1}-Y_{2}\right)^{2} / n\right)^{1 / 2}$, onde $Y_{1}$ é o valor do $V O_{2 \text { máx }}$ predito por cada teste de campo, $Y_{2}$ é o valor da medida direta obtida pelo método de referência e n é o número de sujeitos. Foi calculado também o coeficiente de variação (CV) pela divisão do erro padrão de estimativa pela média do $\mathrm{VO}_{2}$ estimado pelos testes de campo, multiplicado por 100.

Medidas de concordância entre os escores de $\mathrm{VO}_{2 \text { máx }}$ estimados pelos testes de campo COOPER, MILHA e SR-20M e a medida direta obtida em teste de laboratório foram adotadas, de acordo com os procedimentos descritos na literatura ${ }^{21}$, incluindo os limites de concordância de 95\% e análise de tendência.

O nível de significância estatística adotado foi de $P<0,05$. As análises foram conduzidas utilizando-se os pacotes estatísticos SPSS for Windows, versão 17.0, e o Med Calc for Windows, versão 4.15.

\section{RESULTADOS}

A descrição das características físicas dos sujeitos é apresentada na tabela 1, por meio de valores de média, desvio padrão, valores mínimos e máximos, referentes às variáveis: idade, massa corporal, estatura, índice de massa corporal e somatória de dobras cutâneas.

As comparações entre os escores de $\mathrm{VO}_{2 \text { máx }}$ estimados pelos testes de campo COOPER, MILHA e SR-20M e a medida direta obtida em teste de laboratório são apresentadas na tabela 2. Os resultados indicaram que o teste de COOPER e MILHA superestimaram (0,9 e 6,9\%, respectivamente) e o teste SR-20M subestimou (8,5\%) os valores relativos de $\mathrm{VO}_{2 \text { máx }}$ quando comparados ao método de referência, embora nenhuma diferença estatisticamente significante tenha sido verificada $(P>0,05)$.

Os critérios de desempenho dos testes de campo COOPER, MILHA e SR-20M são ilustrados na tabela 3. A reta estabelecida pelo modelo de regressão linear apresentou informações semelhantes para os três testes de campo. Os valores de intercepto não foram considerados diferentes do ponto zero, embora os valores de slope a partir da estimativa do $\mathrm{VO}_{2 \text { máx }}$ pelos três testes de campo tenham sido diferentes ( $P$ $<0,05)$ do valor um, a partir da reta de identidade. Os testes COOPER, MILHA e SR-20M como variáveis preditoras do $\mathrm{VO}_{2 \text { máx }}$ explicaram 37, 41 e $38 \%$ da variância, respectivamente, da medida direta do $\mathrm{VO}_{2 \text { máx }}$. Os EPE e o EP foram relativamente baixos (aproximadamente 6\%) e semelhantes para cada teste.

Tabela 1. Características gerais da amostra $(n=12)$.

\begin{tabular}{c|c|c|c|c}
\hline Variáveis & Média & DP & Mínimo & Máximo \\
\hline Idade (anos) & 23,1 & 2,8 & 20 & 31 \\
\hline Massa corporal $(\mathrm{kg})$ & 68,7 & 12,1 & 51,6 & 90,9 \\
\hline Estatura $(\mathrm{cm})$ & 173,2 & 9,9 & 161,2 & 196,5 \\
\hline IMC $\left(\mathrm{kg} / \mathrm{m}^{2}\right)$ & 22,7 & 2,2 & 19,3 & 26,1 \\
\hline$\Sigma 7 D C(\mathrm{~mm})$ & 115,7 & 33,1 & 67 & 169 \\
\hline
\end{tabular}

Nota: $\mathrm{DP}=$ desvio padrão; $\mathrm{IMC}$ = índice de massa corporal; $\Sigma 7 \mathrm{DC}$ = somatório de sete dobras cutâneas.

Tabela 2. Comparação entre valores diretos de $\mathrm{VO}_{2 \text { máx }}\left(\mathrm{ml} \cdot \mathrm{kg}^{-1} \cdot \mathrm{min}^{-1}\right)$ e estimativas obtidas por meio dos testes COOPER, MILHA e SR-20M.

\begin{tabular}{c|c|c|c|c}
\hline & Média & DP & Mínimo & Máximo \\
\hline MD & 42,2 & 7,3 & 29,8 & 52,9 \\
\hline COOPER & 42,6 & 6,6 & 32,6 & 54,6 \\
\hline MILHA & 45,1 & 4,5 & 36,1 & 50,1 \\
\hline SR-20M & 38,6 & 4,4 & 32,6 & 44,6 \\
\hline
\end{tabular}

Nota. $\mathrm{DP}$ = desvio padrão; $\mathrm{MD}$ = teste direto; COOPER = teste de corrida/caminhada de 12 minutos; MILHA = teste de corrida/caminhada de uma milha; SR-20M = teste vai-e-vem de $20 \mathrm{~m}$. Nenhuma diferença estatisticamente significante $(P>0,05)$ quando comparados com MD. 
A figura 1 ( $A, B, C)$ ilustra a plotagem proposta por Bland e Altman ${ }^{21}$ para verificação dos limites de concordância entre a medida do $\mathrm{VO}_{2 \operatorname{máx}}$ obtida pelo método de referência e pelos testes de campo COOPER (A), MILHA (B) e SR-20m (C). A disposição gráfica dos resultados apresentou limites de concordância na ordem de $-0,4 \pm 12,0 \mathrm{ml}^{\mathrm{kg}}{ }^{-1} \cdot \mathrm{min}^{-1}$ entre MD e COOPER (A), sem indicativo de tendência na variabilidade individual $(r=0,12 ; P=0,70)$. Por outro lado, os testes da MILHA (B) e SR-20M (C) tiveram os limites de concordância inferiores, com valores de $-2,8$ $\pm 11,0$ e $3,6 \pm 11,3 \mathrm{ml} \cdot \mathrm{kg}^{-1} \cdot \mathrm{min}^{-1}$, respectivamente, e semelhante valor de análise de tendência $(r=0,55 ; P=0,06)$ que, da mesma forma, não detectou associação entre a média e a diferença do método direto contra as estimativas fornecidas pelos testes de campo.

Tabela 3. Critérios de desempenho: intercepto, inclinação, coeficiente de correlação $(R)$, erro padrão de estimativa (EPE), erro puro (EP), coeficiente de variação (CV) e concordância (viés, limites e tendência) entre o $\mathrm{VO}_{2}$ mensurado pelo método de direto e estimado pelos três testes de campo COOPER, MILHA e SR-20m ( $n=12)$.

\begin{tabular}{c|c|c|c|c|c|c|c|c|c}
\hline & \multirow{2}{*}{ Intercepto } & Inclinação & $\mathbf{R}$ & EPE & EP & CV & \multicolumn{3}{|c}{ Concordância } \\
\cline { 6 - 10 } & & & & & & Viés & Limites & Tendência \\
\hline COOPER & 13,32 & $0,68^{b}$ & 0,61 & 6,03 & 5,89 & 14,14 & $-0,4$ & 11,$6 ;-12,4$ & 0,71 \\
\hline MILHA & $-4,82$ & $1,04^{b}$ & 0,64 & 5,85 & 6,05 & 12,98 & $-2,8$ & 8,$1 ;-13,8$ & 0,06 \\
\hline SR-20m & 3,01 & $1,02^{b}$ & 0,62 & 6,02 & 6,59 & 15,59 & 3,6 & 14,$9 ;-7,6$ & 0,06 \\
\hline
\end{tabular}

Nota: $\mathrm{COOPER}=\mathrm{VO}_{2}$ estimado pelo teste de corrida/caminhada de $12 \mathrm{~min} ; \mathrm{MILHA}=\mathrm{VO}_{2}$ estimado pelo teste de corrida/caminhada de uma milha; $\mathrm{SR}-20 \mathrm{~m}=\mathrm{VO}_{2}$ estimado pelo teste shuttle run de $20 \mathrm{~m} .{ }^{\mathrm{b}}=$ diferente de $1, P<0,05$.

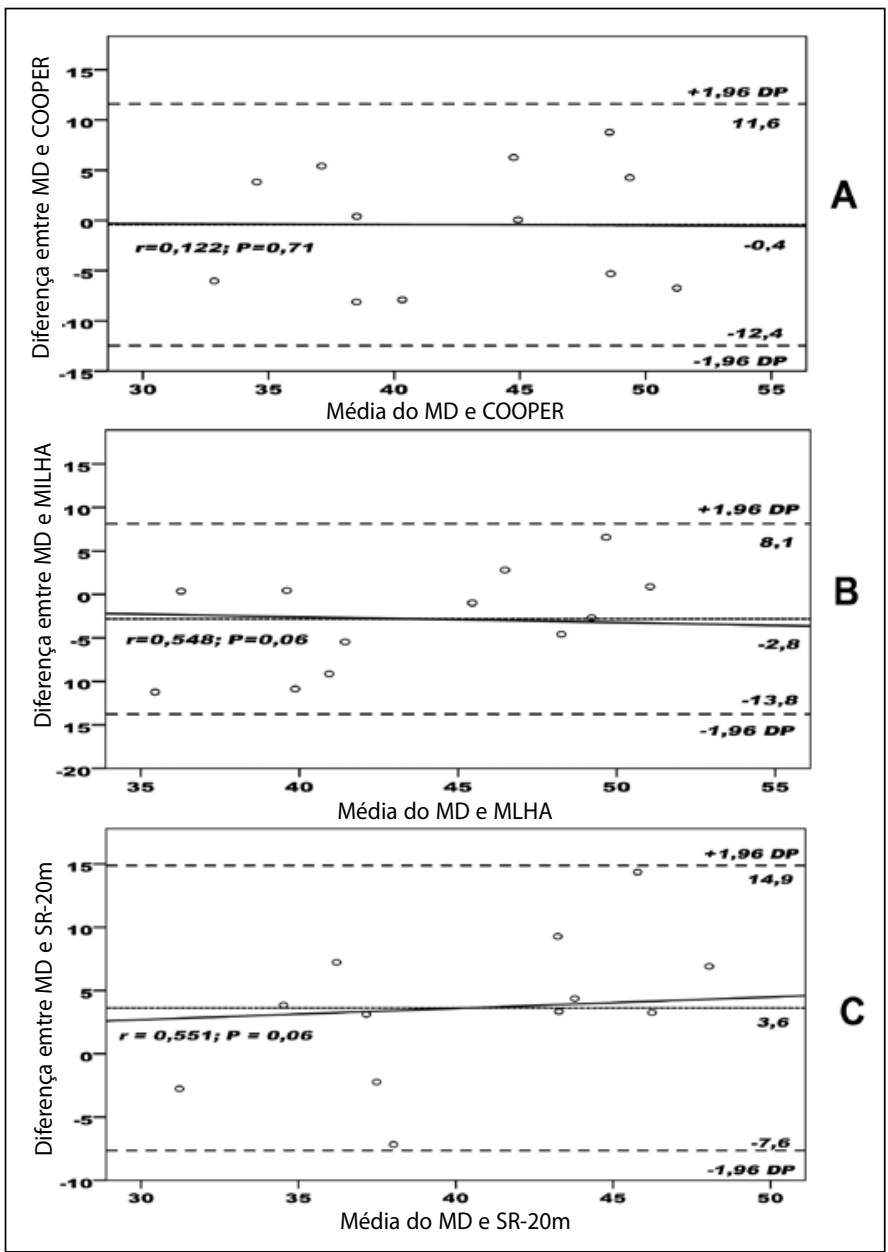

Figura 1. Limites de concordância e análise de tendência entre os valores diretos de $V_{O_{2 m a ́ x}}(\mathrm{ml} . \mathrm{kg}-1$.min-1) (MD) e estimativas obtidas por meio dos testes COOPER (A), MILHA (B) e SR-20M (C) $(n=12)$.

\section{DISCUSSÃO}

O presente estudo comparou o $\mathrm{VO}_{2 \text { máx }}$ de jovens universitários estimado por três diferentes testes de campo com o $\mathrm{VO}_{2 \text { máx }}$ obtido pelo método de referência (MD). Além disso, a concordância e a validade dos testes e suas respectivas equações de predição por meio de recursos estatísticos específicos para este fim, também, foram investigadas. Tais análises consistem na verificação dos parâmetros de validação considerando os valores coletivos e individuais, por meio de comparações de médias, análise de regressão simples, avaliação dos erros e coeficientes de variação gerados por cada equação, limites de concordância (95\%) e medida de tendência. As principais informações produzidas por este estudo são discutidas a seguir.

\section{Validade do teste de corrida/caminhada de 12 minutos (COOPER)}

Embora nenhuma diferença estatisticamente significante tenha sido encontrada na comparação entre as médias de $\mathrm{VO}_{2 \text { máx }}$ mensuradas pelo MD e estimadas pelo teste de COOPER $(P>0,05)$, as medidas obtidas a partir do teste COOPER foram superestimadas em cerca de $0,9 \%$. Esse valor confirma as informações relatadas por Grant et al. ${ }^{12}$, em uma amostra de homens jovens saudáveis de 22 anos.

Os parâmetros obtidos pela análise de desempenho do teste de COOPER forneceram valores de r, EPE, EP e CV na ordem de 0,61, 6,03, 5,89 e 14\%, respectivamente (tabela 3). Assim, apesar de os valores médios serem similares àqueles identificados pelo $\mathrm{MD}$, uma correlação apenas moderada foi encontrada, com valores inferiores aos relatados em outros estudos ${ }^{12,13,22}$. Entretanto, esse tipo de análise, de forma isolada, não parece ser suficiente para considerar um teste de campo válido para determinada população, visto que os coeficientes de correlação simples indicam apenas a força da relação entre as duas medidas, considerando valores de tendência central. Desse modo, não é possível estabelecer a amplitude dos erros na estimativa do $\mathrm{VO}_{2 \text { máx }}$ nem analisar a existência ou não de concordância entre os valores gerados individualmente.

Diante disso, a análise dos limites de concordância entre a medida padrão e as estimativas obtidas pelos testes de campo foi realizada, além da utilização desta técnica para verificar a possibilidade de tendência de medida. Assim, de acordo com as informações apresentadas pela plotagem de Bland e Altman ${ }^{21}$, a expectativa quando administramos um teste de campo em situações semelhantes ao teste direto com os mesmos sujeitos é de que as diferenças médias entre os escores devem se aproximar do valor zero e os limites extremos dos intervalos de confiança (95\%) devem se aproximar ao máximo dos valores das diferenças médias.

O teste de COOPER (figura 1A), apresentou viés de medida na ordem de $-0,4 \pm 12,0 \mathrm{ml} . \mathrm{kg}^{-1}$. $\mathrm{min}^{-1}$, sem indicativo de tendência na variabilidade individual $(r=0,12 ; P=0,70)$. A amplitude nos limites de concordância foi de certa forma preocupante e demonstrou uma variabilidade individual entre os resultados que não foi possível identificar apenas pelos procedimentos de comparação e desempenho. Assim, o teste de COOPER poderia subestimar os valores de $\mathrm{VO}_{2 \text { máx }} \mathrm{em}$ $11,6 \mathrm{ml} \cdot \mathrm{kg}^{-1} \cdot \mathrm{min}^{-1}(27,7 \%)$ e superestimar em 12,4 ml. $\mathrm{kg}^{-1} \cdot \mathrm{min}^{-1}(29,4 \%)$. Em termos estatísticos, podemos afirmar que, em $95 \%$ dos casos, o mesmo indivíduo submetido ao teste MD e ao teste de campo COOPER pode apresentar amplitude de variação nas medidas por volta dos 12,0 ml.kg-1 min $^{-1}$.

Os valores encontrados neste estudo se diferem em parte daqueles relatados em investigação relativamente semelhante por Penry et al.22, em uma amostra de homens e mulheres de 18 a 33 anos $(n=21)$. Os autores verificaram menores valores de 
subestimação (3,8\%), menor amplitude de variação nas medidas $\left(8,5 \mathrm{ml} \cdot \mathrm{kg}^{-1} \cdot \mathrm{min}^{-1}\right)$ e maiores valores de correlação $(r=0,87)$ quando comparados aos desta investigação. Entretanto, uma tendência de estimativa foi identificada apontando que o teste de COOPER parece subestimar o $\mathrm{VO}_{2 \text { máx }}$ dos sujeitos menos aptos e superestimar os valores daqueles com melhor condicionamento aeróbio. Vale destacar que, diferente dos resultados obtidos por Penry et al.22, no presente trabalho o teste de COOPER não apresentou tendência na estimativa $(r=0,12 ; P=0,70)$, o que demonstra que este teste avalia os indivíduos da mesma forma, independente de o $\mathrm{VO}_{2 \text { máx }}$ ser baixo ou alto. Este fato, se confirmado por outras investigações, pode representar uma vantagem interessante para a utilização deste teste, visto que o teste de COOPER passaria a ser uma boa alternativa para avaliação de amostras consideradas heterogêneas quanto à condição cardiorrespiratória.

\section{Validade do teste de corrida/caminhada de uma milha (MILHA)}

O $\mathrm{VO}_{2 \text { máx }}$ estimado pelo teste de campo da MILHA, com a utilização da equação proposta por Margaria et al. ${ }^{19}$ para conversão do tempo total no teste em $\mathrm{VO}_{2 \text { máx, }}$ superestimou em 6,9\% os valores médios obtidos pelo MD. Apesar de os valores encontrados indicarem um maior percentual de superestimação do $\mathrm{VO}_{2 \text { máx }}$ quando comparado ao observado no teste de COOPER nas comparações frente ao MD, o teste da MILHA foi, entre os testes analisados neste estudo, aquele que apresentou o maior valor de correlação com $M D(r=0,64)$ e coeficiente de explicação mais elevado $\left(R^{2}=0,41\right)$, além do menor EPE $\left(5,85 \mathrm{ml} \cdot \mathrm{kg}^{-1} \cdot \mathrm{min}^{-1}\right)$ e a menor variação dos valores em relação à média ( $C V=12,98 \%)$.

As informações de desempenho do teste da MILHA e a respectiva equação na estimativa do $\mathrm{VO}_{2 \text { máx }}$ obtidas no presente estudo podem ser consideradas semelhantes a um trabalho realizado com objetivo de criar e validar uma equação para o teste da MILHA. O estudo conduzido por Cureton et al. ${ }^{23}$ propôs uma equação generalizada para estimativa do $\mathrm{VO}_{2 \text { máx }}$ de homens e mulheres de oito a 25 anos de idade. Os resultados indicaram EPE próximo a 4,8 $\mathrm{ml} . \mathrm{kg}^{-1} \cdot \mathrm{min}^{-1}$, bem como coeficientes de explicação por volta de $R^{2}=0,50$, que foram considerados válidos. Entretanto, nenhum tipo de análise foi utilizada para análise da concordância entre o valor real de $\mathrm{VO}_{2 \text { máx }} \mathrm{e}$ as estimativas obtidas pela equação proposta, limitando os resultados apenas à análise do grupo.

No Brasil, um trabalho recente ${ }^{24}$ procurou investigar a validade da equação proposta por Cureton et al. ${ }^{23}$ em uma amostra de homens jovens, fisicamente ativos, adotando a análise de Bland e Altman ${ }^{21}$ para verificação da concordância entre as medidas. Os resultados demonstraram que o teste da MILHA, juntamente com a equação proposta por Cureton et al. ${ }^{23}$, subestimaram os valores de $\mathrm{VO}_{2 \text { máx }}$ em 11,8\%, além de apresentar baixa correlação $(r=0,21)$ e amplos limites de concordância $\left(-5,9 \pm 14,7 \mathrm{ml} \cdot \mathrm{kg}^{-1} \cdot \mathrm{min}^{-1}\right)$ entre a medida padrão e a estimativa pelo teste da MILHA.

No presente estudo, contudo, os valores de $\mathrm{VO}_{2 \text { máx }}$ estimados pela equação proposta por Margaria et al. ${ }^{19}$ para o teste de MILHA indicaram menor viés de medida $\left(-2,8 \pm 11,0 \mathrm{ml} \cdot \mathrm{kg}^{-1} \cdot \mathrm{min}^{-1}\right)$ em relação aos estudos supracitados e sem tendência na estimativa $(r=0,55 ; P=0,06)$. Assim, na amostra utilizada o teste da MILHA refletiu de maneira mais adequada os parâmetros do grupo.

Uma possível explicação para as diferenças encontradas, pode estar atrelada, pelo menos em parte, ao fato de que equações de predição têm melhores informações de validade quando aplicadas em populações com características semelhantes às das amostras utilizadas para o desenvolvimento das equações.

\section{Validade do teste de corrida de vai-e-vem de 20 m (SR-20M)}

O teste de corrida vai-e-vem ou shuttle run de 20 m (SR-20m) é um dos testes de campo mais utilizados para estimativa do $\mathrm{VO}_{2 \text { máx }} \mathrm{e}$ diagnóstico da aptidão cardiorrespiratória em diferentes populações. Proposto por Léger e Lambert ${ }^{20}$, o teste SR-20m proporciona a estimativa do $\mathrm{VO}_{2 \text { máx }}$ a partir de diferentes equações ${ }^{11}$ desenvolvidas, especificamente, para jovens e adultos.

No presente estudo, os resultados não apontaram diferença entre a média fornecida pelo método de referência e a estimativa obtida pelo teste SR-20m, utilizando-se a equação proposta por Léger et al. ${ }^{11}$ para adultos. Ao contrário do que foi verificado no teste de COOPER e MILHA, os valores médios de $\mathrm{VO}_{2 \text { máx }}$ foram subestimados em 8,5\% pelo teste SR-20m. Outros estudos disponíveis na literatura já haviam relatado resultados relativamente semelhantes, com os valores médios de $\mathrm{VO}_{2 \text { máx }}$ sendo subestimados pelo teste SR-20m de 1 a 10\% 8,10-14,22,25-29.

Os critérios de desempenho utilizados neste estudo para análise do teste SR-20m indicaram respostas bastante similares àquelas observadas nos testes de COOPER e MILHA, com EPE e EP de aproximadamente $6,0 \mathrm{ml} \cdot \mathrm{kg}^{-1} \cdot \mathrm{min}^{-1}$ e capacidade de explicar a variabilidade nos valores de $\mathrm{VO}_{2 \text { máx }}$ obtidos pelo $\mathrm{MD}$ em $38 \%$. Além disso, os valores de concordância encontrados foram na ordem de 3,6 $\pm 11,3 \mathrm{ml} . \mathrm{kg}^{-1}$. min $^{-1}$. Tal como verificado no teste da MILHA, os valores estiveram próximos do estabelecimento de uma tendência na estimativa do $\mathrm{VO}_{2 \text { máx }}(r=0,55 ; P=0,06)$.

Dentre os poucos trabalhos que analisaram medidas de concordância para análise da reprodutibilidade e validade do teste SR-20m, Cooper et al. ${ }^{28}$ relataram, em estudo conduzido com homens universitários ativos, valores de reprodutibilidade elevados $(r=0,98)$, com viés nas medidas de concordância $\left(1,8 \pm 6,3 \mathrm{ml} \cdot \mathrm{kg}^{-1} \cdot \mathrm{min}^{-1} ; P=0,004\right)$. Os valores médios de $\mathrm{VO}_{2 \text { máx }}$ foram subestimados em cerca de $3,1 \%$, mas sem confirmação de tendência $(r=0,08 ; P=0,66)$, assim como no presente estudo.

Análises semelhantes às anteriormente citadas também foram estabelecidas em uma amostra de homens adultos saudáveis $(n=74)$ por Metsios et al. ${ }^{29}$. Os pesquisadores verificaram a validade e reprodutibilidade do teste SR-20m, obtendo coeficiente de correlação bastante semelhante ao encontrado no presente estudo ( $r=0,63$ vs. 0,62, respectivamente); contudo, os limites de concordância $\left(0,6 \pm 6,8 \mathrm{ml} \cdot \mathrm{kg}^{-1} \cdot \mathrm{min}^{-1}\right)$ e coeficiente de variação ( $C V=6,8 \%)$ foram inferiores aos encontrados nesta investigação.

Em estudo com homens e mulheres, Penry et al. ${ }^{22}$ verificaram a validade de dois testes de campo, entre eles o SR-20m em sujeitos de 18-33 anos. A análise de concordância demonstrou amplitude de variação na estimativa por volta de $7,0 \mathrm{ml} \cdot \mathrm{kg}^{-1} \cdot \mathrm{min}^{-1}$, mas sem tendência de medida. Diferente do presente estudo, os autores encontraram importantes diferenças, com os valores médios de $\mathrm{VO}_{2 \text { máx }}$ medidos pelo método de referência, sendo subestimados em aproximadamente 6\% pelo teste SR-20m $(P<0,05)$.

Apesar do tamanho da amostra reduzido, da variabilidade quanto aos níveis de aptidão física dos indivíduos e do fato de as análises terem sido conduzidas sem a estratificação por sexo, os procedimentos estatísticos utilizados permitiram análise mais detalhada de desempenho e concordância, proporcionando uma interpretação mais rigorosa acerca das informações produzidas sobre cada um dos testes investigados. Diante dos resultados observados, recomenda-se que sejam realizadas outras investigações envolvendo testes de campo para avaliação da potência aeróbia, controlando alguns fatores como o sexo, o nível de aptidão física dos indivíduos, bem como as possíveis diferenças morfológicas associadas ao estado nutricional e nível de atividade física habitual. 


\section{CONCLUSÕES}

Os resultados do presente estudo sugerem que os valores médios de $\mathrm{VO}_{2 \text { máx }}$ estimados pelos testes de campo COOPER, MILHA e SR-20m em indivíduos adultos jovens parecem relativamente similares aos obtidos pelo método direto. Por outro lado, a análise de concordância adotada demonstrou amplos limites de variação entre as medidas, para os três testes. Assim, de maneira geral, os testes de campo analisados podem ser utilizados como uma boa alternativa para análise dos níveis de aptidão cardiorrespiratória, destacando que o teste de COOPER parece ser o mais interessante para fornecer parâmetros individuais em amostras mais heterogêneas, enquanto que os testes de MILHA e SR-20m podem fornecer melhores indicativos do comportamento de grupos de sujeitos.

\section{AGRADECIMENTOS}

Os autores agradecem ao Conselho Nacional de Desenvolvimento Científico e Tecnológico (CNPq) pelas bolsas de produtividade em pesquisa (E.R.V.R e E.S.C.) e a Coordenação de Aperfeiçoamento de Pessoal de Nível Superior (CAPES) pela bolsa de Doutorado (M.B.B. e V.F.M.) outorgadas.

Todos os autores declararam não haver qualquer potencial conflito de interesses referente a este artigo.

\section{REFERÊNCIAS}

1. Blair SN, Kohl HW 3rd, Paffenbarger RS Jr, Clark DG, Cooper KH, Gibbons LW. Physical fitness and all-cause mortality. A prospective study of healthy men and women. JAMA 1989;262:2395-401.

2. Lamonte MJ, Barlow CE, Jurga R, Kampert JB, Church TS, Blair SN. Cardiorespiratory fitness is inversely associated with the incidence of metabolic syndrome: a prospective study of men and women. Circulation 2005;112:505-12

3. Lee D, Artero EG, Sui X, Blair SN. Mortality trends in the general population: the importance of cardiorespiratory fitness. J Psychopharmacology 2010;24;Supp4: 27-35.

4. Astrand PO. Experimental studies of physical work capacity in relation to sex and age. Copenhagen: Ejnar Munksgaard; 1952.

5. Basset DR Jr, Howley ET. Limiting factors for maximum oxygen uptake and determinants of endurance performance. Med Sci Sports Exerc 2000;32:70-84.

6. Bassett DR Jr, Howley ET, Thompson DL, King GA, Strath SJ, McLaughlin JE, et al. Validity of inspiratory and expiratory methods of measuring gas exchange with a computerized system. J Appl Physiol 2001;91:218-24.

7. Cyrino ES, Okano AH, Silva KES, Altimari LR, Dórea VR, Zucas SM, et al. Aptidão aeróbia e sua relação com os processos de crescimento e maturação. Rev Educ Fís/UEM 2002;13:17-26.

8. Grant JA, Joseph AN, Campagna PD. The prediction of $\mathrm{VO}_{2}$ max: a comparison of 7 indirect tests of aerobic power. J Strength Cond Res 1999;13:356-62.

9. Cyrino ES, Papst RR, Altimari LR, Okano AH, Caldeira LFS, Gobbo LA, et al. Comparação entre a potência aeróbia estimada por dois testes de campo. Rev Educ Fís/UEM 2005;16:171-7.

10. Stickland MK, Petersen SR, Bouffard M. Prediction of maximal aerobic power from the 20-m multi-stage shuttle run test. Can J Appl Physiol 2003;28:272-82.

11. Léger LA, Mercier D, Gadoury C, Lambert J. The multistage 20-meter shuttle run test for aerobic fitness. J Sports Sci 1988;6:93-101.

12. Grant S, Corbett K, Amjad AM, Wilson J, Aitchison T. A comparison of methods of predicting maximum oxygen uptake. Br J Sports Med 1995;29:147-52

13. McNaughton L, Hall P, Cooley D. Validation of several methods of estimating maximal oxygen uptake in young men. Percept Mot Skills 1998;87:575-84.

14. Duarte MFS, Duarte CR. Validade do teste aeróbico de corrida de vai-e-vem de 20 metros. Rev Bras Ciên Mov 2001;9:7-14

15. Atkinson G, Nevill AM. Statistical methods for assessing measurement error (reliability) in variables relevant to sports medicine. Sports Med 1998;26:217-38.
16. Gordon CC, Chumlea WC, Roche AF. Stature, recumbent length, and weight. In: Lohman TG, Roche AF, Martorell R, editors. Anthropometric standardization reference manual. Champaign: Human Kinetics Books; 1988

17. Harrison GG, Buskirk ER, Carter LE, Johnston FE, Lohman TG, Pollock ML, et al. Skinfold thicknesses and measurement technique. In: Lohman TG, Roche AF, Martorell R, editors. Anthropometric standardization reference manual. Champaign: Human Kinetics Books; 1988.

18. Cooper KH. A means of assessing maximal oxygen uptake. JAMA 1968;203:135-8.

19. Margaria R, Aghemo P, Limas FP. A simple relation between performance in running and maximal aerobic power. J Appl Physiol 1975;38:351-2.

20. Léger L, Lambert J. A maximal multistage 20-m shuttle run test to predict $\mathrm{VO}_{2}$ max. Eur J App Physiol 1982;49:1-12.

21. Bland JM, Altman DG. Statistical methods for assessing agreement between two methods for clinical measurement. Lancet 1986:8:307-10.

22. Penry JT, Wilcox AR, Yun, J. Validity and reliability analysis of Cooper's 12-minute run and the multistage shuttle run in healthy adults. J Strength Cond Res 2011;25:597-605.

23. Cureton KJ, Sloniger MA, O'Bannon JP, Black DM, McCormack WP. A generalized equation for prediction of $\mathrm{VO}_{2}$ peak from 1-mile run/walk performance. Med Sci Sports Exerc 1995;27:445-51.

24. Almeida JA, Campbell CSG, Pardono E, Sotero RC, Magalhães G, Simões HG. Validade de equações de predição em estimar $\mathrm{O} \mathrm{VO}_{2}$ max de brasileiros jovens a partir do desempenho em corrida de $1.600 \mathrm{~m}$. Rev Bras Med Esporte 2010;16:57-60.

25. Ramsbottom R, Brewer J, Williams C. A progressive shuttle run test to estimate maximal oxygen uptake Br J Sports Med 1988;22:141-4.

26. Ahmaidi S, Collomp C, Caillaud C, Préfaut C. Maximal and functional aerobic capacity as assessed by two graduated field methods in comparison to laboratory exercise testing moderately trained subjects. Int J Sports Med 1992;13:243-8.

27. Sproule J, Kunalan C, McNeill, Wright H. Validity of 20-MST for predicting $\mathrm{VO}_{2}$ max of adult Singaporean athletes. Br J Sports Med 1993;27:202-4.

28. Cooper SM, Baker JS, Tong RJ, Roberts E, Hanford M. The repeatability and criterion related validity of the $20 \mathrm{~m}$ multistage fitness test as a predictor of maximal oxygen uptake in active young men. $\mathrm{Br} J$ Sports Med 2005;39:1-7.

29. Metsios GS, Flouris AD, Koutedakis Y, Nevill A. Criterion-related validity and test-retest reliability of the 20m square shuttle test. J Sci Med Sport 2008;11:214-7. 